\title{
Richard GOULET (éd.), Dictionnaire des philosophes antiques, Va : de Paccius à Plotin; Vb : de Plotina à Rutilius Rufus
}

Pierre Pontier

\section{(C) OpenEdition}

1 Journals

\section{Édition électronique}

URL : https://journals.openedition.org/philosant/825

DOI : 10.4000/philosant.825

ISSN : 2648-2789

Éditeur

Éditions Vrin

\section{Édition imprimée}

Date de publication : 1 novembre 2014

Pagination : 321-322

ISBN : 978-2-7574-0855-1

ISSN : 1634-4561

\section{Référence électronique}

Pierre Pontier, « Richard goulet (éd.), Dictionnaire des philosophes antiques, Va : de Paccius à Plotin ; Vb : de Plotina à Rutilius Rufus », Philosophie antique [En ligne], 14 | 2014, mis en ligne le 01 novembre 2018, consulté le 02 décembre 2022. URL : http://journals.openedition.org/philosant/825 ; DOI : https://doi.org/10.4000/philosant.825

\section{(c)}

Creative Commons - Attribution - Pas d'Utilisation Commerciale - Pas de Modification 4.0 International - CC BY-NC-ND 4.0

https://creativecommons.org/licenses/by-nc-nd/4.0/ 


\section{COMPTES RENDUS}

Richard Goulet (éd.), Dictionnaire des philosophes antiques, Va: de Paccius à Plotin; Vb : de Plotina à Rutilius Rufus, Paris, CNRS Éditions, 2012, 2 vol., 1885 pages, ISBN Va : 978-2-271-07335-8; Vb : 978-2-271-07399-0.

Avec un certain humour noir, R. Goulet a mis en exergue de son avant-propos un proverbe arabe : «Lorsque le Tout-Puissant - loué soit-Il - estime que l'éternité ne sera pas suffisamment longue pour châtier un pécheur, Il lui inspire de commencer sur terre un dictionnaire. » On mesure avec respect la justesse de la citation à la lecture du substantiel cinquième et avant-dernier tome du Dictionnaire des philosophes antiques qu'il fait paraître trente ans après la mise en œuvre du projet. En raison du nombre et de l'importance des philosophes dont le nom commence par $\mathrm{P}$, ce tome présente la particularité d'être divisé en deux volumes, Va et $\mathrm{Vb}$, à la pagination continue (1883 pages, autrement dit en moyenne le double des tomes précédents; on comprend les raisons éditoriales d'une telle division, même si cela rend la consultation des index un peu plus difficile). De fait, aux quatre notices (Platon, Plotin, Porphyre et Proclus) qui ont une longueur légitime de plus de deux cents pages, s'ajoute la notice consacrée à Plutarque de Chéronée d'une longueur supérieure à cent pages. Les notices sur Plotin, Porphyre et Proclus témoignent notamment des recherches importantes faites dans ce domaine philosophique depuis une vingtaine d'années. L'entreprise monumentale qui a sollicité comme toujours les meilleurs spécialistes reste fidèle à son esprit originel, autrement dit une conception large et érudite du philosophe, inspirée par l'enseignement de P. Hadot à la mémoire duquel ce volume est logiquement dédié, d'où la présence dans ce tome comme dans les autres de personnes dont le lien avec la philosophie peut parfois paraître relativement ténu : c'est ici le cas de poètes tels que Plaute ou d'hommes politiques tels que Pompée. Mais on ne saurait critiquer dans ce domaine une recherche d'exhaustivité toujours scientifiquement fondée.

L'autre parti pris du dictionnaire est la liberté relative laissée aux auteurs sur laquelle Richard Goulet s'était expliqué dans le tome 1 (p. 21) : elle a pour conséquence des variations dans la forme de rédaction adoptée, la longueur des notices et le style (qui va du plus elliptique à l'exposé ou à la dissertation). C'est particulièrement clair dans la notice consacrée à Platon, dont les contributeurs sont souvent ceux qui ont traduit les dialogues dans la collection Garnier-Flammarion autour de Luc Brisson (à l'exception notamment du Gorgias, du Ménon, du Cratyle, du Sophiste, du Politique et du Philèbe) : en fonction des auteurs, certains dialogues sont traités de façon plus ou moins approfondie et selon des approches différentes. Le lecteur, averti, sait de toute façon à quoi s'en tenir ; et il en allait de même jadis pour les articles de la Realencyclopaedie dont le Dictionnaire des philosophes antiques se rapproche dans la démarche, avec ses fins propres, et à laquelle il fait toujours référence. Enfin, d'un point de vue formel, et quelle que soit l'« appréhension » de l'éditeur (p. 9), le Dictionnaire est impeccablement édité (on aura seulement noté

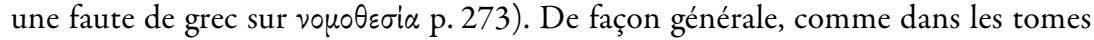


précédents, une grande attention est accordée à la réception : une vingtaine de pages fort intéressantes sont consacrées à Platon et la tradition arabe, et l'on trouve même une rubrique sur Plutarque et la culture européenne.

À une époque où les projets à court terme sont privilégiés, un tel travail collectif d'érudition mené sur une trentaine d'années n'obtiendrait pas actuellement de financement et il ne pourrait pas voir le jour. C'est pourtant sans doute l'une des publications les plus largement utiles, pour l'étudiant comme pour le chercheur. On n'en est que plus admiratif devant la somme de travail réalisée par le maître d'œuvre et ses contributeurs, et l'on se sent redevable à leur égard d'avoir mis à notre disposition un outil de travail infiniment précieux.

Pierre PONTIER

Université Paris-Sorbonne Paris 4

Magali ANNÉE, Parménide: Fragments Poème, précédé de Énoncer le verbe être, Paris, Librairie philosophique J. Vrin, 2012 (Bibliothèque des textes philosophiques), 215 pages, ISBN 978-2-6116-2414-0.

Une étude qui se situe exclusivement sur le terrain linguistique est bien placée pour attirer l'attention de l'interprète de Parménide, avide d'un éclairage nouveau sur un texte tellement commenté qu'il semble ne plus être possible d'y apporter quoi que ce soit. Le point de vue du grammairien pourrait en effet révéler certaines subtilités jusque-là ignorées, en particulier s'il ne cherche pas à mettre la grammaire au service d'une signification philosophique (comme on l'a déjà fait abondamment) mais à limiter la signification à la grammaire même. Autrement dit : que peut-on tirer de la lettre d'un texte avant d'y introduire toute notion héritée de l'histoire de la pensée ? Cependant, pour mener à bien cet exercice, il n'était pas nécessaire de discréditer le point de vue philosophique en prétendant que toute interprétation du poème en termes de conceptualisation du verbe être ou d'étude de sa signification est anachronique car influencée par les conceptions de l'être développées plus tard par Platon et Aristote (p. 11-12). S'il est clair que la langue poétique de Parménide est caractéristique de l'époque archaïque, pourquoi faudrait-il en déduire que toute distinction de significations, d'emplois et de fonctions du verbe être serait chez lui inadéquate (p. 42) ? Ce présupposé, selon lequel tant le philosophe que le poète est entièrement tributaire de l'expression de son époque et incapable d'innover par rapport à elle, reste ininterrogé et injustifié, ainsi que la question de savoir quelles sont alors les conditions d'évolution d'une langue et d'une pensée.

La thèse de l'ouvrage est donc que Parménide est avant tout un poète qui joue avec la langue, qui exploite certains procédés linguistiques pour mettre en évidence l'importance syntaxique et morphologique du verbe «être ». Toute la première partie du poème, jusqu'au fragment 8 inclus, a une fonction introductive et exhortative, destinée à montrer que la polysémie de ce verbe et ses « variations paradigmatiques sans limites » sont indispensables pour comprendre le véritable contenu du poème, c'est-à-dire la partie cosmologique, et pour « assurer une assise infaillible à la parole même du poète-savant $\gg($ p. $41 ; i d$. p. 59). Ainsi, la revendication d'une vérité persuasive du discours ne repose pas sur la validité des démonstrations, 